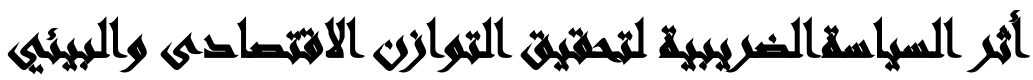

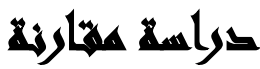

\section{[17]}

عمرو حسين عبد البر(') - وائل فوزى عبد الباسط(')- مروة أحمد عبد الحافظ

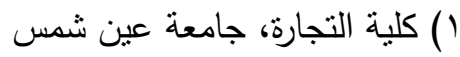

\section{المهريله}

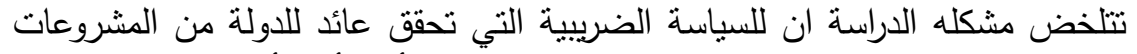

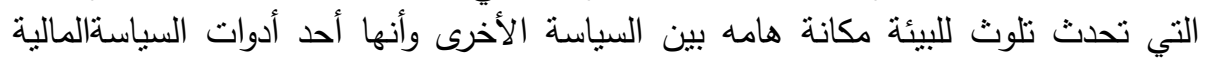

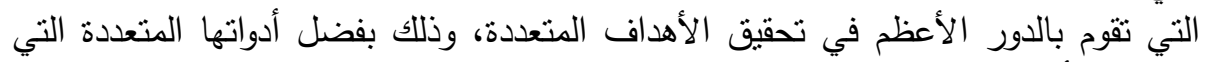

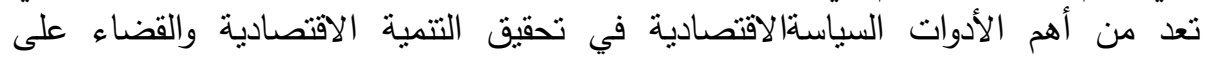

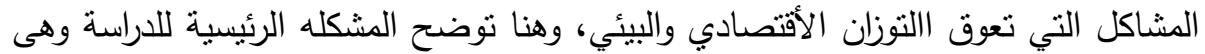

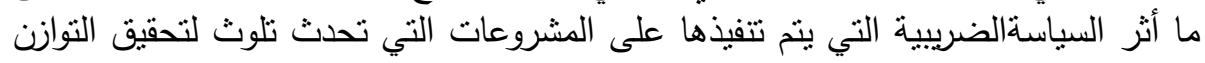
الأقتصادي والبيئي.

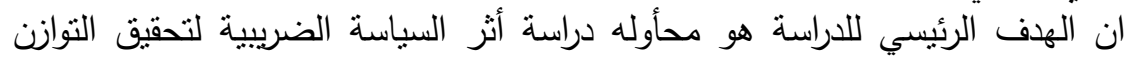

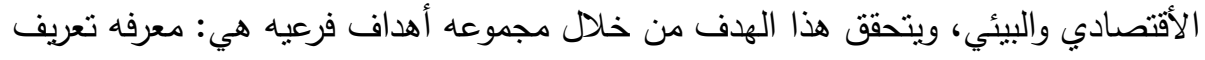

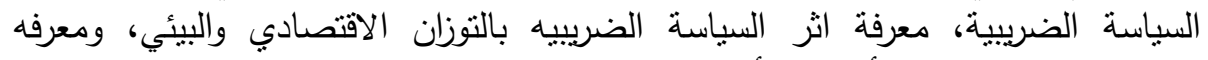
الاعفاءات الضربيية وما أهميتها وأنواعها.

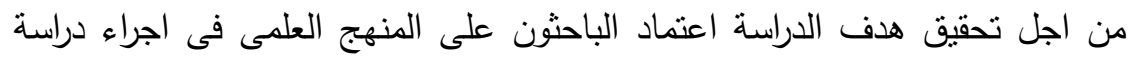

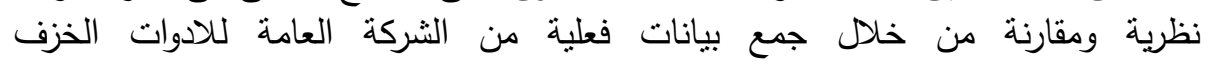
والصيني(مصنع الادوات الصحية)، وذلك من اجل اختبار فروض الدانة فلدراسة وتحليل البيانات وتقسير النتائج.

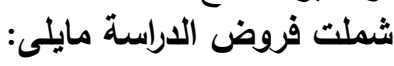
الفرض الاول: نوجد علاقة جوهرية ذاتدلاله معله معوية بين السياسة الضريبية والتوازن الاقتصادي.

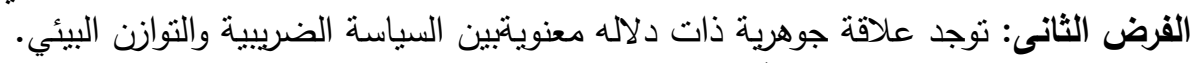
قا تم التوصيل الى النتائج التالية:

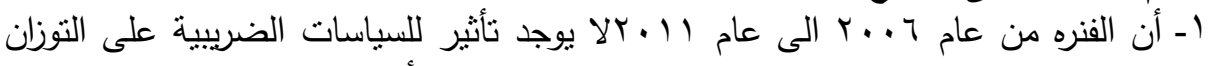

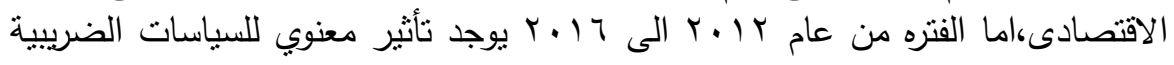

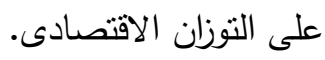




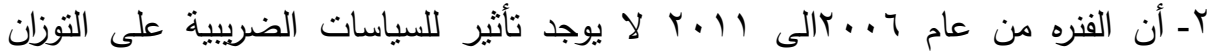

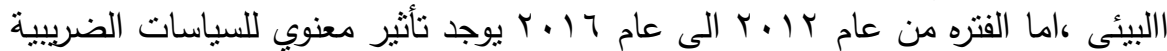

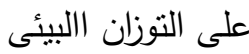

وفى ضوء النتائج السابقة قد أوصت الدراسة بمجموعة من التوصيات ومن أهمها مايلى:

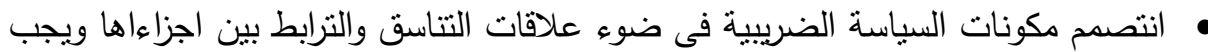
أن توازن بين العدالة والفعالية والبساطة.

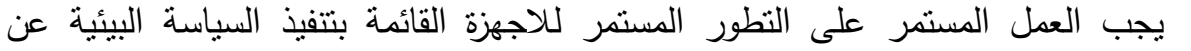
طريق تدريب العاملين بها وتوفير الاجهزة والمعدات للازمة للقيام باعمالها.

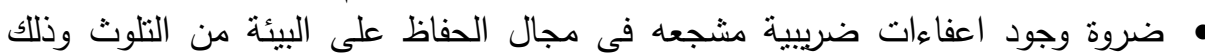

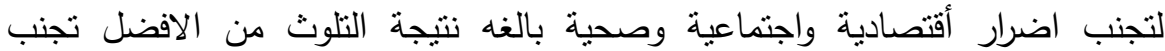
المشكلة قبل وقوعها اى تجنب التلوث قدر الامكانوذلك للتشجيع للحفاظ على البيئة.

\section{xasall}

لا شك أن السياسة الضريبية لها إنعكاسات هامه على النشاط الاقتصادي باعتبار أن

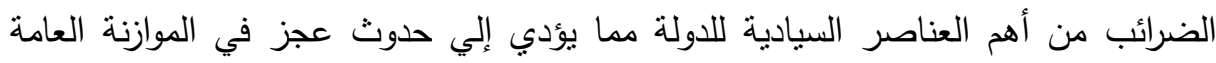

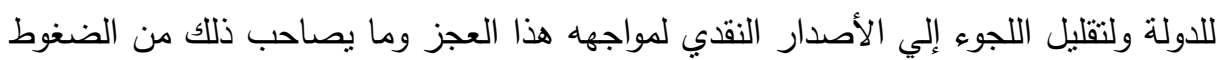

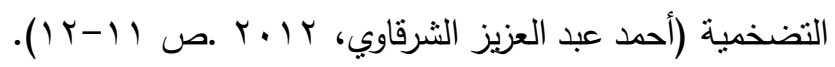

أن السياسة الضريبية ماهى الإ جزء من السياسة الاقتصادية للدولة وإن النظام الضريبي

ليس فقط أداة من أدوات السياسة الضريبية بل هو ترجمة عملية لتلك السياسة فإن تحقيق التهن

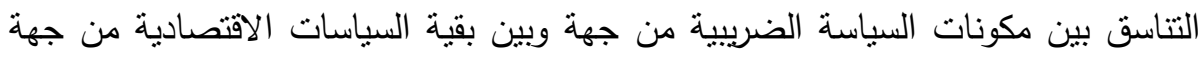
أخرى ويعد من الأمور الضرورية والحتمية لتحقيق النجاح للسياسة في تحقيق أهدافها (عنايات

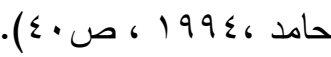

انها اليوم تلعب دورا هام فى جميع النواحي الاقتصادية والأجتماعية والسياسية، وكما

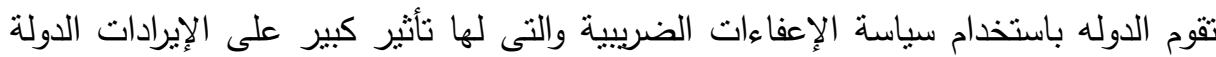
وتقوم الدولة بمنح الأعفاءات الضريبية للشركات الملوثة للبيئة ومن أجل حماية البيئة وتحقيق الإني التوزان البيئى وإيضا من أجل تحقيق التوزان الاقتصادي عن طريق تنأوى الكمية المعروضة التهنة من الإنتاج مع الكمية المطلوبة من الإنتاج، لم يكن هناك تعريف محدد للإعفاءات الضريبية 
وذلك لإتساع مفهومه وتطوره وأغراضه وتتوع أثنكاله أدى إلي صعوبة حصره في تعريف محدد

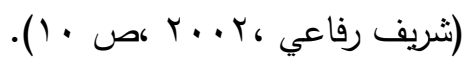

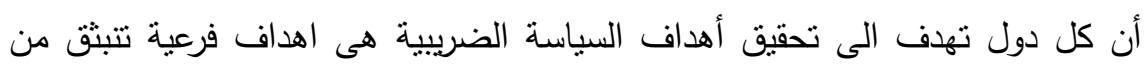

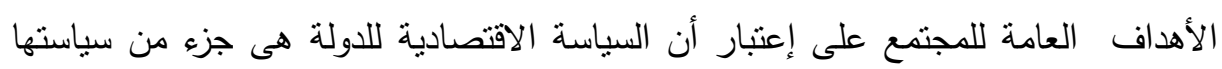

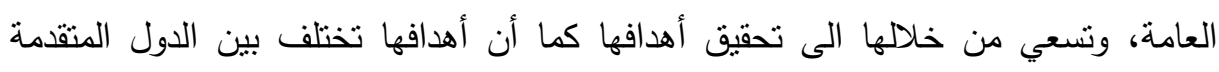

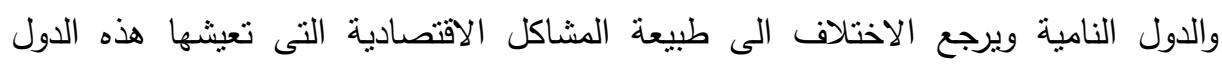

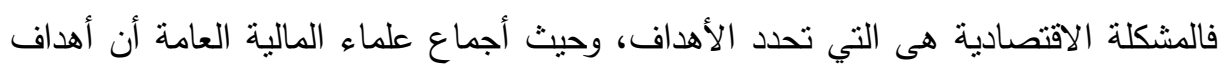

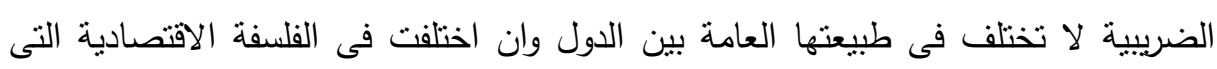
تؤمن بها، وأختلفت فى درجة تقدمها الاقتصادى ( Monzer Kanf , 1997, p147) مع ذلك تطور النشاط الأقتصادي و أنساع رقعتة، و أعتماد فكر العولمة الاقتصادية القادية

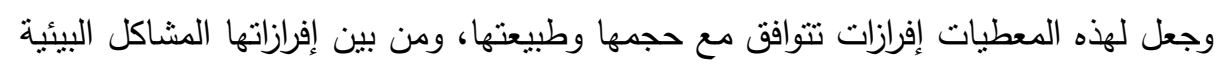

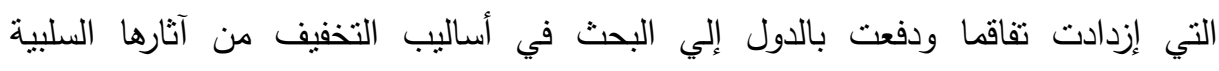
.http://mawdoo3.com

إن حماية البيئة واجب كل أنسان لأن المجتمع الراقي هو الذي يحافظ على بيئته

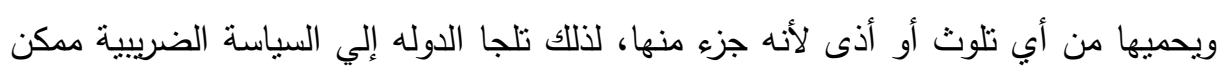

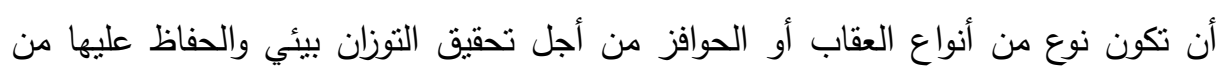

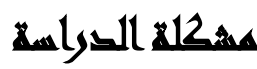

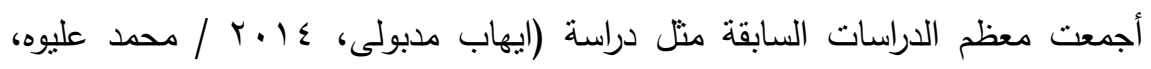

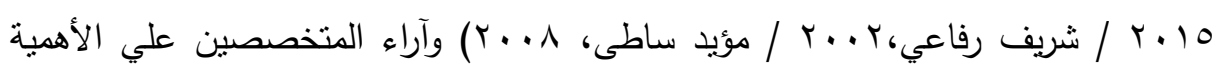
الاقتصادية والبيئية من أستخدام سياسات لضريبية من خلال أستخدام سياسة الإعفاءات الضربيية لتحقيق التوزان الأقتصادي والبيئي.

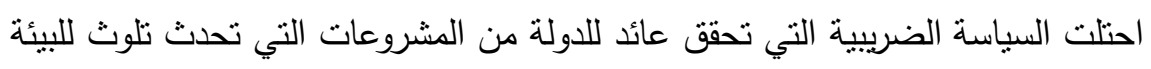

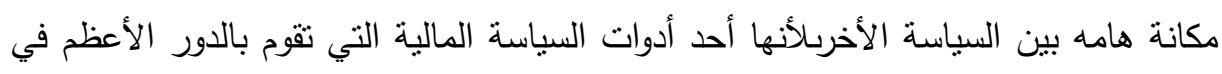

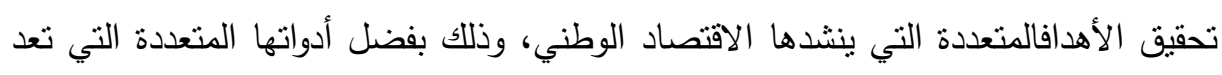

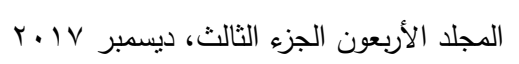


من أهم الأدوات السياسة الاقتصادية في تحقيق التتمية الاقتصادية والقضاء علي المشاكل

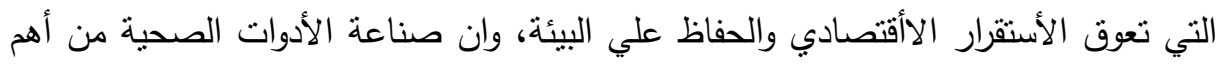
وأخطر الصناعات ثاثيراً علي البيئة، وتتمنل فى ظهور الأنبعاثات فى الهواء والتربة نتيجة

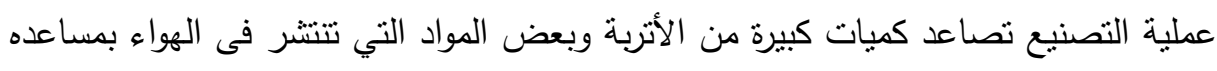
العوامل الطبيعية منل الرياح وكذلك أيضا ينتج مخالفات صلبة تؤثر علي التربة، وهذه التئه

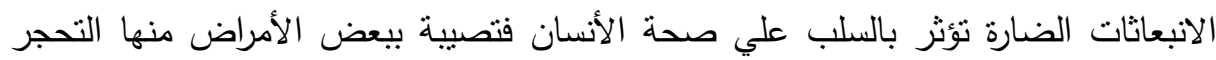
الرئه والالتهاب الرئوي والأمراض الجلدية والنفسية وأمراض العيون الناتجة من الاتربة، وتؤثثر علي حاسة السمع لدي العاملين بسب التعرض المستمر فى الضوضاء الناتج من الكسارات.

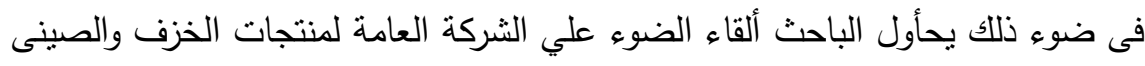
(مصنع الأدوات الصحية) وتأثر السياسة الضريبية عليه لتحقيق التوزان الأقتصادي والتوزان أسيكلة المهيد

تسعي الدراسة الي الإجابة علي التساؤلات التالية: () ما المقصود بالسياسة الضريبية ؟

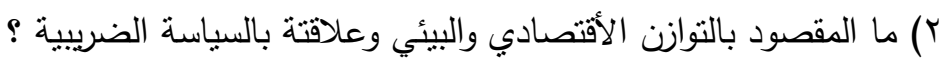

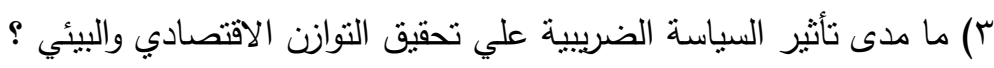

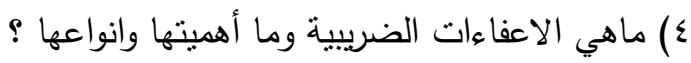

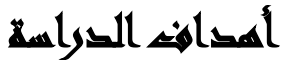

الهدف الرئيسي للبحث هو دراسة أثز السياسة الضريبية لتحقيق التوازن الاقتصادي

$$
\text { والبيئي ويتحقق هذا الهدف من خلال مجموعه أهداف فرعي: }
$$

1 - معرفة المقصود بالسياسية الضريبية. r- معرفة المقصود بالتوازن الاقتصادي والبيئي وعلاقه ذلك بالسياسة الضريبية.

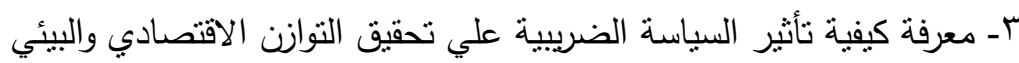
ع - معرفه المقصود بالاعفاءات الضريبية وما أهميتها وانواعها. 


\section{أهمية التصواسمة}

تتمثل أهية هذه الدراسة من الناحيتين العلمية والعملية: الناحية العلمية: أن السياسة الضريبية دورا فعال فى تحقيق النتزان الاقتصادي والبيئي وذلك عن طريق استخدام سياسة الإعفاءات الضريبية واعفاء بعض المشروعات الملوثة للبيئة من الضرائب مقابل الحفاظ علي البيئة وتحقيق التوزان الاقتصادى.

الناحية العملية: تقييم التجربة المصرية لتحقيق التوزان الاقتصادي والتوزان البيئي علي الثركة العامه للحزف والصينى (مصنع الأدوات الصحية).

\section{مبوض القواسم}

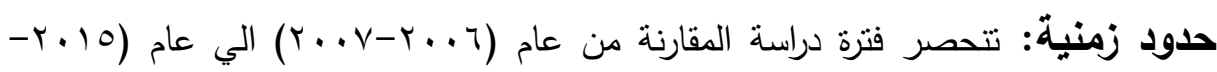
$\cdot(r .17$ حدود مكانية: نشمل نطبيق الدراسة علي الثركة العامة للخزف والصينى( مصنع الأدوات (الصحة )

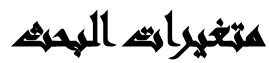

المتغير المستقل: السياسة الضريبية المتغير التابع الاول: التوازن الاقتصادي ويمكن قياسة عن طريق تساوى الكمية المعروضة مع الكمية المطلوبة. المتغير التابع الثانى: التوزان البيئى ويكن قياسة عن طريق بيانات الصحية للعاملين قبل العمل فى المصنع وبعد العمل وطرق حماية البيئة من الاضرار البيئية.

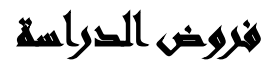

الفرض الأول: توجد علاقة جوهرية ذات دلاله معنوية بين السياسة الضريبية والتوازن الاقتصادي. الفرض الثاني: توجد علاقة جوهرية ذات دلاله معنوية بين السياسة الضريبية والتوازن البيئي.

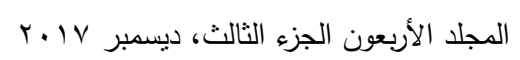




\section{التواسايت المربعية}

تضمنت الدراسة العديد من الدراسات المرجعية التي ناقتت وتتأولت موضوع الدراسة وفيما يلي أهم هذه الدراسات مرتبة ترنيبا زمنيا:

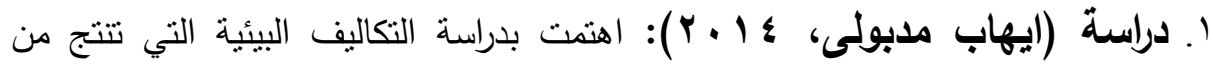
الأثار البيئية الناجمة من مصنع السيراميك فى مصر ، وحيث تتتج عن التوسع فى صناعة السيراميك العديد من الأثارالسلبية التي تؤثز علي البيئة منل الانبعانات الغازية والأتربة والمخلفات الصلبة التي تؤثز علي عملية التتمية، ويدرس البحث كيفية مواجهة هذه لهنه الأثارالسلبية والعمل علي منعها أو الحد منها وذلك لتعظيم العائد البيئي. Y.دراسة (محمد عليوه، 1 م ب Y): اهتمت بالقاء الضوء علي مشكلات التلوث الناجمة

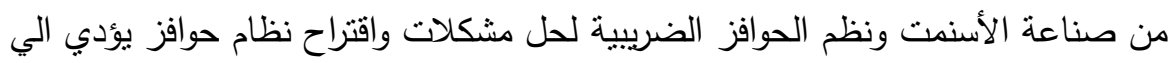
تحقيق التوزان بين الأهمية الاقتصادية لمصانع الأسمنت وأثارها الاقتصادية والأجنماعية والبيئية ومما يحقق من عائد اقتصادى ومالى واجتماعي وبيئى للجنمع وذلك من خلال:

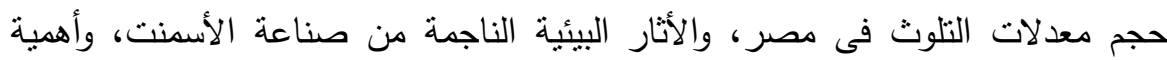

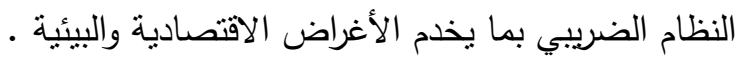
r. دراسة (شريف رفاعي، + . r): اهتمت بدراسة بتحديد و مدى دور الإعفاءات الضريبية فى أتخاذ قرارات الأستثمار وكما تتمثل أيضا اهميتها فى ربط النظم الضريبية بسياسة الاستثمار بربط مباشر وفعال فالنظم الضريبية نسعى لي تشجيع المستثمر علي توظيف أمواله فى تحقيق خطه التتمية الاقتصادية والأجتماعية للاولة وهى بذلك تاكد بكائ

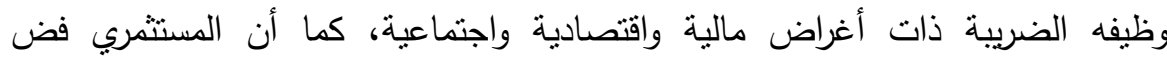
المعامله المتميزة التي تتيح له الفرصة الحصول علي أكبر قدر ممكن من عائد مزواله النشاط الاستثمارى، وبذلك الحصول علي عده إعفاءات ضريبية التي يترتب عليها تمتعة لهن بسعر ضريبي مميزه أو تخفيض وباء الوعاء الضرائب أو إعفاء منها مطلق أو محدود . 


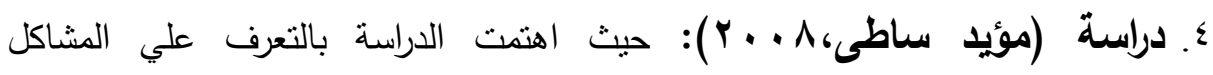
الاقتصادية المختلفة واليات معالجتها بأستخدام السياسة الضربيية والإعفاءات الضريبية التي تستخدمها الدولة، وأهمية موضوع بالنسبة للقطاع المحلى والأجنبى الأستثمارى لفهم

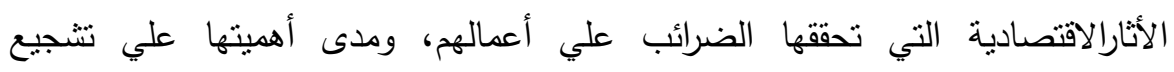

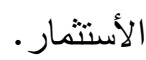

\section{الإطالر النظليه اللتراسة}

- تعرف السياسة المالية: علي أنها استخدام الدولة لإيراداتها ونفقاتها بما يحقق أهدافها الاقتصادية والأجتماعية والسياسية في ظل ما تعتتقه من عقائد في حدود إمكانيات المتاحة مع الأخذ في الاعتبار درجة تقدمها ونموها الاقتصادي. - تعريف الضريبة: هي مبلغ من المال تفرضه الدولة علي المكلفين باعتبارهم أعضاء متضامنين في منظمة سياسية مشتركة هي الدولة بهدف تأمين الخدمات العامة والإصلاح

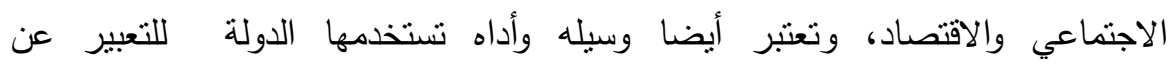

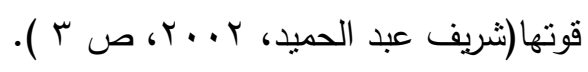

- النظام الضريبي: هو عباره عن التشريعات التي تفرض أوضاعا معينة متعلقة بتحديد اشخاص الملتزمين بأداء الضريبة مع بيان النظام وكيفية قياس المادة الخاضعة للضريبة حتى يمكن تعين القدر المالى من هذا الألنزام تمهيداً لأتخاذ إجراءات تحصيلة وتمويل فيلة الفيل

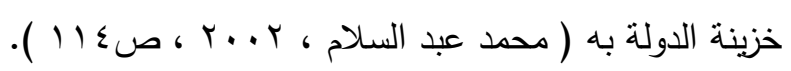


أولا: تعريف السياسة الضريبية: رغم الأهمية المتزايده للضريبة فى الحياه الاقتصادية

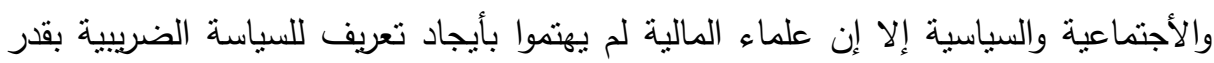

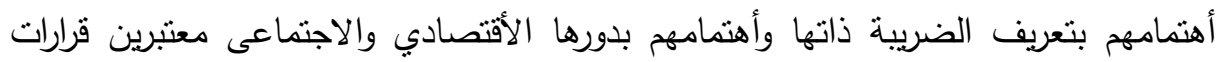
الدولة بشان الضرائب هى سياسة الدولة الضريبية لا يعنى ذلك عدم وجود نعريف للسياسة الضريبية حيت تتعدد التعريفات ومنها:

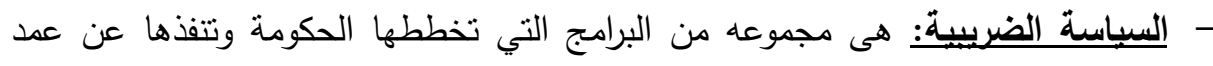
مستخدمه فيها كافه الأدوات الضريبية الفعلية والمحتملة لأحداث أثار معينة وتجنب الأثنار

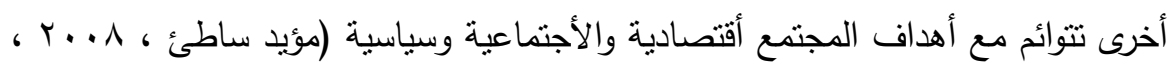

- السباسة الضريية: هى مجموعه متكاملة من أساليب أستخدام الفن الضريبي للإسهام في تحقيق الأهداف، أو مجموعه من البرامج المتكاملة الني تخططها الدولة مستخدمة كافة الته

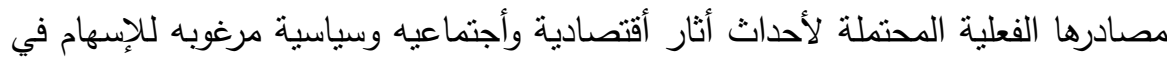

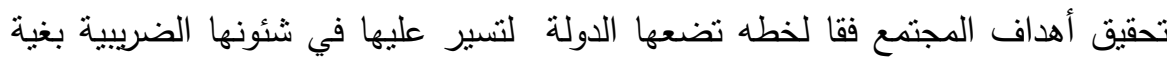
تحقيق الأهداف المالية أو الأجتماعية أو الاقتصادية أو السياسية (عبد العزيز سعيد

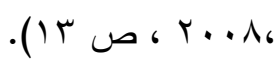
- تعريف السباسة الضريبية الأكثر شمولا: هى مجموعة من البرامج المتكاملة التي تخططها وتتفذها الدولة مستخدمة كافة مصادرها الضريبية الفعلية الاحداث أثنار المرغوبة المبية للمساهمة فى تحقيق أهداف المجتمع (Gravelle Jane, 2014 ,p 6). ثانياً: أهداف السياسة الضريبية: تتعدد الأهداف التي تسعى اليها السياسة الضريبية سواء كانت أهداف أجنماعية أو أقتصادية أو سياسية، ويجب إلإنبان نغالى قى قدرة السياسة الضريبية علي تحقيق الأهداف الاقتصادية (جذب الاستثمار - زيادة الصادرات ) وفى أهداف أجتماعية ممثلة فى اعادة توزيع الدخل، ويجب التركيز علي دعم قدرة السياسة الضريبية علي تحقيق

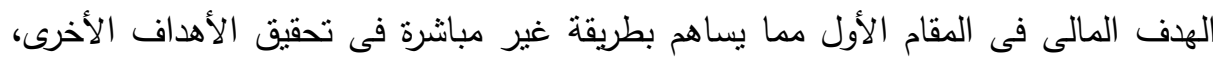


سواء من خلال توفير التمويل للازم أو من خلال التتسيق بين السياسة الضريبية وغيرها من

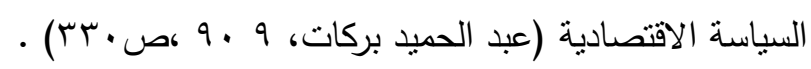

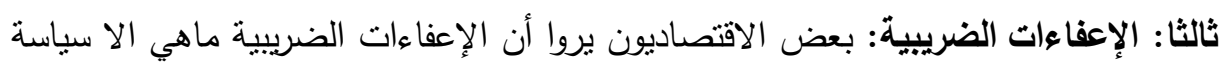

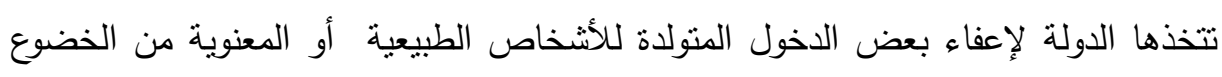

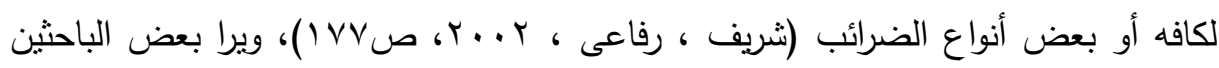

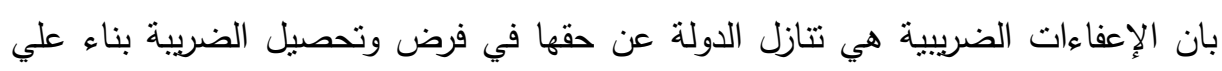

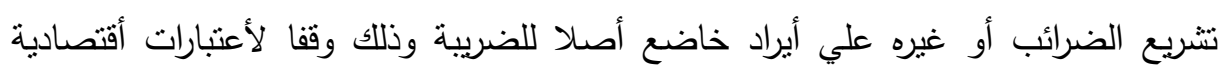

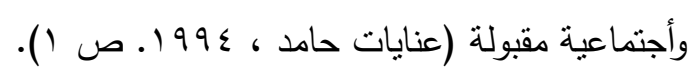
رابعا: أهمية الاعفاعات الضريبية: الإعفاءات الضريبيه لها تأثثر كبير علي الاقتصاد والبيئة البئة

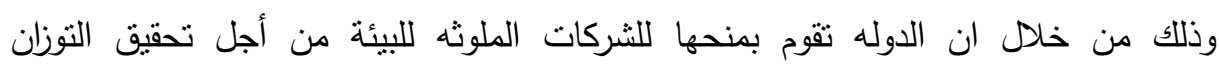
الاقتصادي و البيئي وذللك عن طريق اعطاء اعفاءات ضريبيه للمشروعات الملوثه للبيئة من

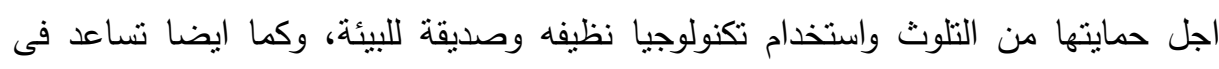
تحقيق التوزان الاقتصادي وذلك عن طريق اعطاء بعض الاعفاءات الضريبيه على ضرائب الارباح من اجل زيادة الاتتاج على السلع المحليه وتخفيض الاسعار تكاليف الانتاج.

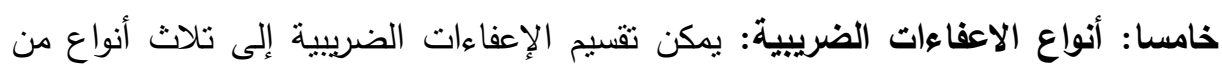

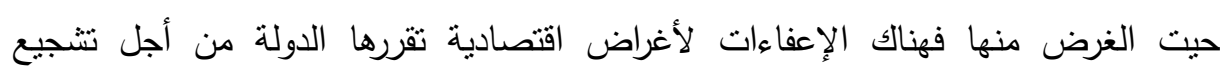
الأستتمار ، والادخار فإن انخفاض الضريبية نتيجة إعفاءات معينة يؤدى إلى زيادة تلقائية في

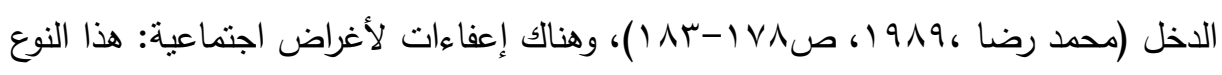

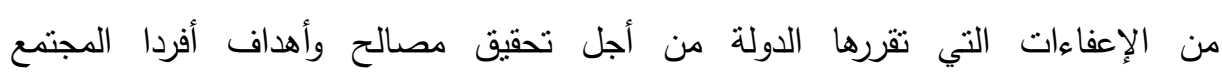

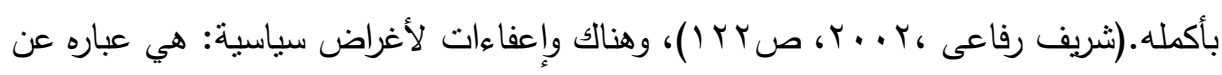

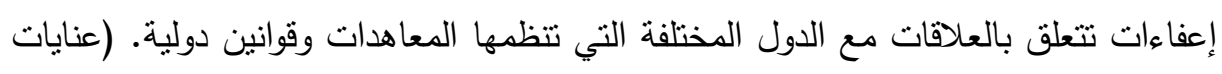

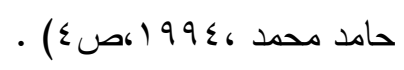


سادسا: أثر السياسة الضريبية على التوزان الاقتصادى: ان السياسة الضريبية ناثير كبيره

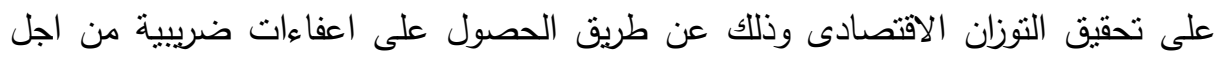

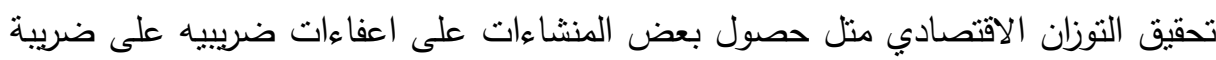

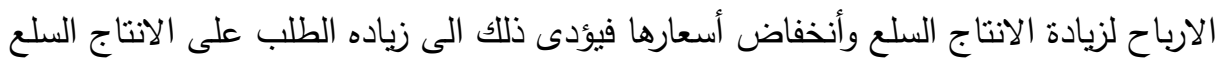

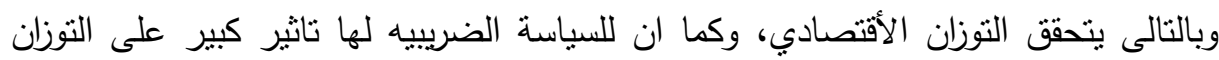

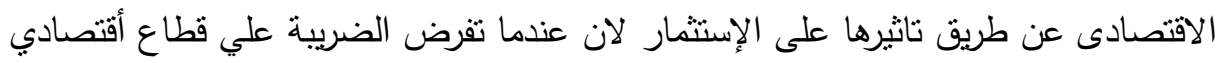

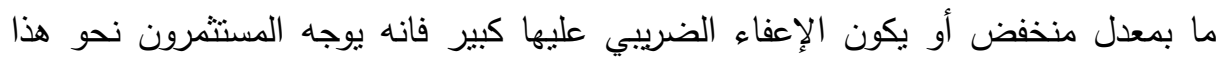

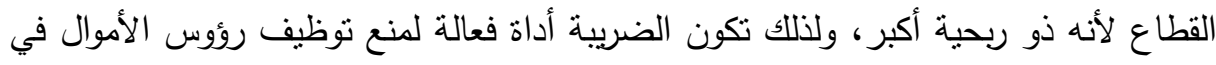
القطاعات الإتتاجية الخاضعة لمعدل ضريبي مرتفع، وكما أن لها تاثير ايضا على الادخار لان عندما تقرض الضريبة على المدخرات يؤدى ذلك إلي تخفيض الدخول المتاحة للإدخار لدي الأفراد، وهذا يعني إنه سيؤدي إلي إنقاص مدخراتهم، ولكن فرض الضرائب علي علي الطبقات

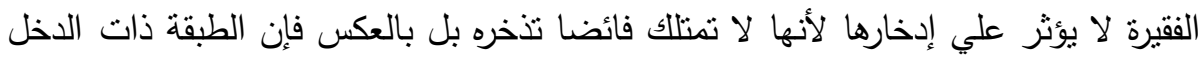

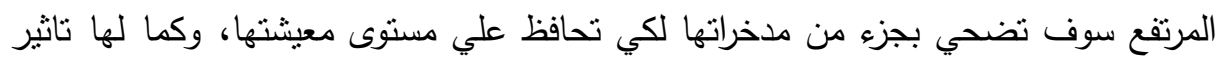

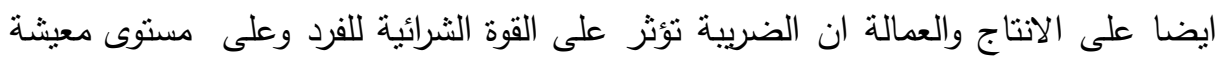

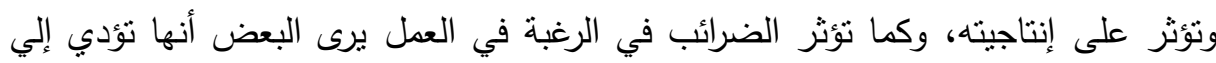

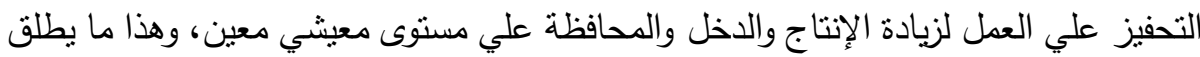
عليه" بالضريبة المحفزة "، وقد تخفض الضرائب على زيادة كميات الإنتاج لانها تزيد من تكلفة معنين الإنتاج وهذا ما يؤدي إلي التقليل من الإنفاق الإستثماري، مما يؤثر علي حجم العمالة في حين

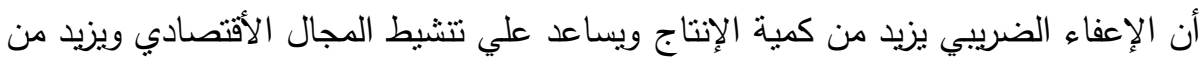

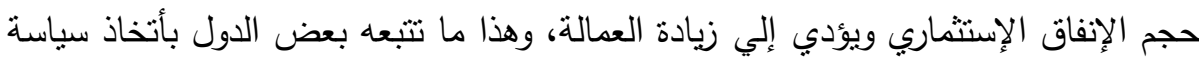
ضريبية من شأنها إعفاء أرباح بعض الإنشطة من الضرائب وذلك للحد من ظاهرة البطالة

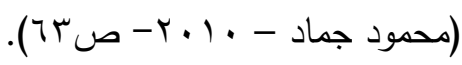




\section{سابعا: التوزان البيئي:}

1 - يعرف تلوث البيئي على أنه هو كل تغير كيفى أو كمى لعناصر ومكونات البيئة يفوق قدره البيئة علي أستيعاب، ومما ينتج عنة أضرار بحياه الأنسان أو قدرتة النظم البيئة علي لني

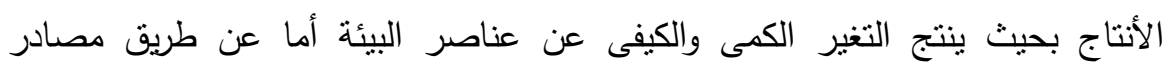
طبيعية دون تدخل الأنسان فى ذلك أو من مصادر صناعية من فعل الأنسان (ايهاب

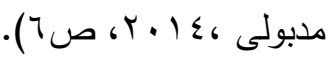

Y- تعريف التوازن البيئي: هو التعادل الطبيعي الذى أوجده الهه سبحانه وتعالى بحكمته وقدرته

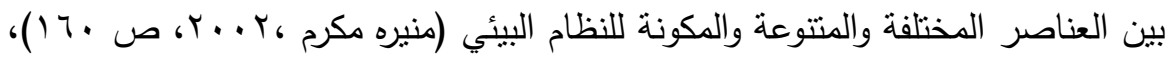
وعلي أنه أيضا بقاء مكونات وعناصر البيئة الطبيعية علي حالتها.

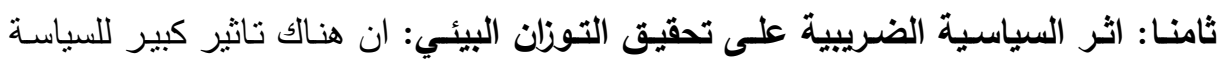
الضريبيه على تحقيق التوزان البيئي وذلك عن طريق: منح إعفاءات ضريبية وحوافز ضريبيه للشركات والمصانع الملوثه للبيئة من اجل استخدام تكنولوجيا صديقة للبيئة حتى تحقق تلك صنك المصانع المستوى منقدم للإلتزام البيئي، وكما يمكن إعطاء هذه المنشآت بطاقات خضراء ينت تجديدها سنويا، تسمح لحامليها بتخفيض الضرائب على المواد المستوردة من الخامات والمواد

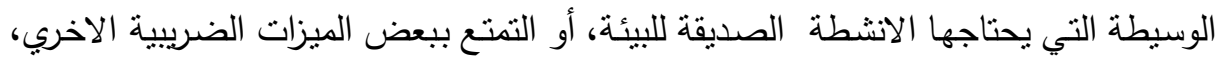
مما يشجع الجميع علي السعي لتحقيق الحدود المطلوبة للمحافظة علي البيئة وتحقيق التوزان البيئي.

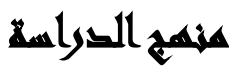

منهج وصفي: تم من خلال تجميع البيانات والمعلومات التي ترتبط بالدراسة عن طريق الأطلاع علي الكتب والدوريات والتقارير والأبحاث والدراسات المنشورة، وأيضاً مطبوعات التهات

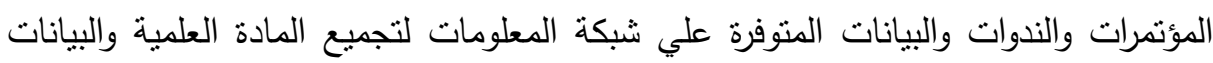
المتاحة عن البحث محل الدراسة. 
منهج استبباطي: تم استخدام التحليل الاستتباطي من حيث استخلاص النتائج وتحليلها،

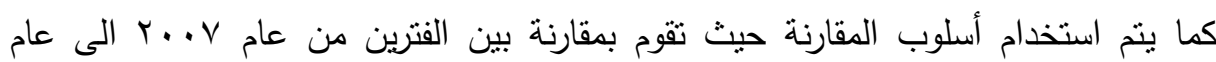

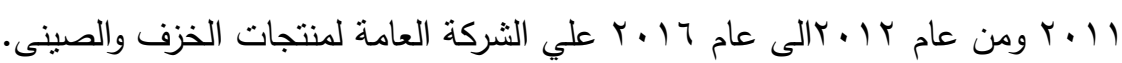

\section{x}

تم تطبيق الدراسة المقارنة على الثركة العامه للمنتجات الخزف والصينى (مصنع

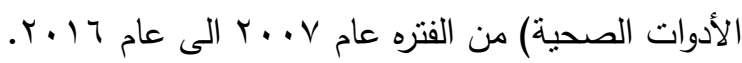

التطبيق الاحصائى لاثبات صحه الفروض: نم تطبيق دراسة مقارنة على الثركة العامه لمنتجات الخزف والصينى (مصنع الادوات الصحية) دراسة مقارنه بين الفترتين من عام

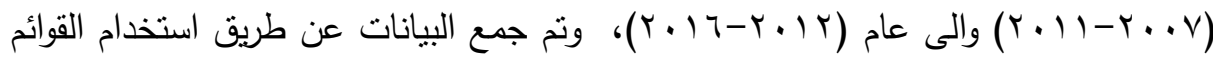

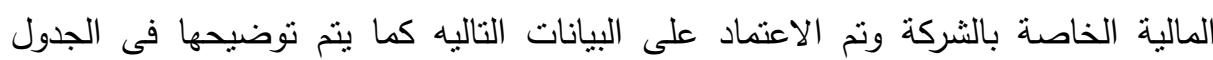
التالي: حان: جدول رقم( (1) : بوضح تعلم طريقة بريل قبل تطبيق البرنامج

\begin{tabular}{|c|c|c|c|c|c|c|c|c|c|}
\hline الدلالة & قيمة Z & قيمة & مجمبوع & متوبط & الالاتحرافيان & المتوسط & المدد & مجموعة المقارنة & اوجه المقارنة \\
\hline \multirow{2}{*}{ غيلزِ } & \multirow{2}{*}{$1 . r 01$} & \multirow{2}{*}{$7 .+$} & YI... & ¿.Y. & OYY.Y. & $\wedge 07 \wedge . \varepsilon$ & 0 & $(Y+11-Y+\cdot V)$ & \multirow{2}{*}{ كمية الاتثاج } \\
\hline & & & $r \varepsilon \ldots$ & 7.A. & $\{\wedge 9 . \wedge)$ & QIr. & 0 & $(Y+17-Y+1 Y)$ & \\
\hline \multirow{2}{*}{ دالة عند } & \multirow{2}{*}{ r.711 } & \multirow{2}{*}{$\cdot . *$} & $10 . .+$ & r... & orl +.9 & $r \leqslant \varepsilon q V . r$ & 0 & $(Y+11-Y+V)$ & \multirow{2}{*}{ قيمة الانتاج } \\
\hline & & & $\varepsilon \ldots$ & A... & orro.7 & HAYI\&.7 & 0 & $(Y+17-Y+1 Y)$ & \\
\hline \multirow{2}{*}{ دالة عند . مند } & \multirow{2}{*}{$1.9 \wedge \varepsilon$} & \multirow{2}{*}{ r.. } & $1 \wedge . \ldots$ & r.7. & IYYE.Y & s & 0 & $(Y+11-Y+V)$ & \multirow{2}{*}{ المحئيةت } \\
\hline & & & rV... & V.E. & VAY.I & IIY.. & 0 & $(Y+17-Y+1 Y)$ & \\
\hline \multirow{2}{*}{ دالة عند } & \multirow{2}{*}{ Y.719 } & \multirow{2}{*}{$\bullet *$} & $10 .+$. & r... & s00H.r & IYА७А.7 & 0 & $(Y+11-Y+V)$ & \multirow{2}{*}{ المحليات } \\
\hline & & & $\varepsilon \ldots+$ & A... & TYQE.T & $r V I 1+.7$ & 0 & $(Y+17-Y+1 Y)$ & \\
\hline & \multirow{2}{*}{$\cdots+$} & \multirow{2}{*}{ IY.0. } & YV.O. & 0.0. &.++ & $1+.+$ & 0 & $(Y+11-Y+V)$ & \multirow{2}{*}{ الضريبية على } \\
\hline & & & YV.O. & 0.0. & $+\ldots$ & $1+.+$ & 0 & $(Y+17-Y+1 Y)$ & \\
\hline \multirow{2}{*}{ فيلز } & \multirow{2}{*}{$1 . r 01$} & \multirow{2}{*}{$7 .+$} & $r \varepsilon \ldots$ & $7 . \wedge+$ & $1+V 7.7$ & rvาl.. & 0 & $(Y+11-Y+\cdot V)$ & \multirow{2}{*}{ التصنية } \\
\hline & & & Y)... & E.Y. & $1+r 0.0$ & YYAI.Y & 0 & $(Y+17-Y+1 Y)$ & \\
\hline \multirow{2}{*}{ مَالِّة } & \multirow{2}{*}{. .OYY } & \multirow{2}{*}{$1+\cdots$} & Yo... & $0 . \ldots$ & YAAY.7 & $1+1+\varepsilon .7$ & 0 & $(Y+11-Y+V)$ & \multirow{2}{*}{ التصنديرات } \\
\hline & & & $r+\ldots$ & $7 .+$. & 19人rr.. & INAYY.E & 0 & $(Y+17-Y+1 Y)$ & \\
\hline \multirow{4}{*}{ غَّلِّ } & \multirow{2}{*}{ מו } & \multirow{2}{*}{ 9... } & rI... & 7.Y. & $1 r \leqslant V .0$ & ror..7 & 0 & $(Y+11-Y+V)$ & \multirow{2}{*}{ قبل الضي الريبية } \\
\hline & & & $r \leqslant \ldots$ & $\varepsilon . \wedge+$ & $17+1.2$ & INVO.A & 0 & $(r+17-Y+1 Y)$ & \\
\hline & \multirow{2}{*}{$1.9 Y \wedge$} & \multirow{2}{*}{$0 .+$} & $Y+\ldots$ & $\varepsilon \ldots$ & $+\ldots$ & $+\ldots$ & 0 & $(Y+11-Y++V)$ & \multirow{2}{*}{ الضريبة } \\
\hline & & & ro.. & V... & 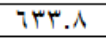 & ¿Or.A. & 0 & $(Y+17-Y+1 Y)$ & \\
\hline & $1.07 \mathrm{~V}$ & $0 . *$ & ro.. & V... & $1+1+.9$ & 9011.7 & 0 & $(Y+11-Y+V)$ & \\
\hline & & & $r+. *$ & $\varepsilon \ldots$ & Yoo.. & $1+V+r . \Lambda$ & 0 & $(Y+17-Y+1 Y)$ & \\
\hline
\end{tabular}


اولا: إختبار صحة فرض الاراسة الاول توجد علاقة بين السياسة الضريبية وتحقيق التوزان

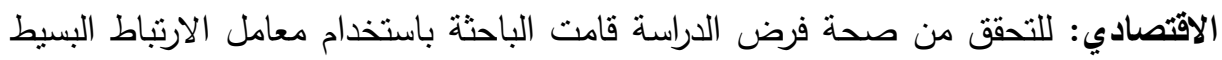
لبيرسون، وتحليل الانحدار البسيط من البط

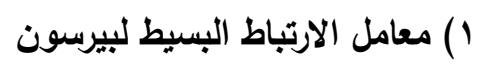

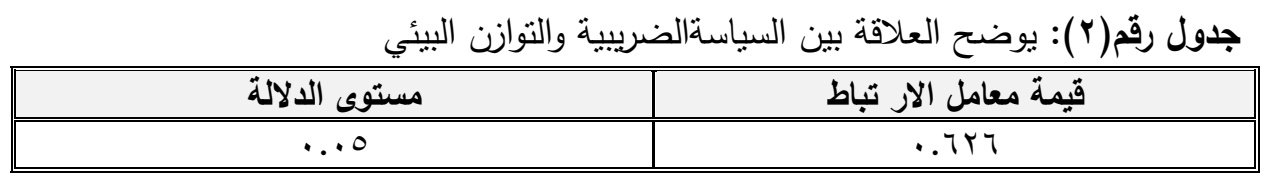

يتضح من بيانات الجدول السابق وجود علاقة أرتباط دالة أحصائياً بين السياسة

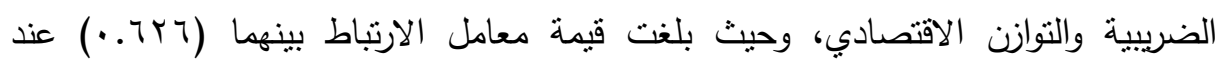

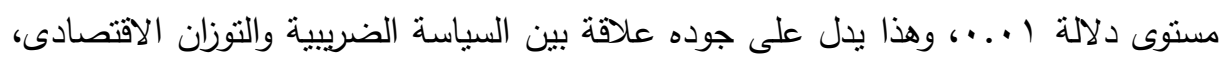
ولذلك هينم عمل تحليل لمعامل الانحدار البسيط لمعرفة نوع العلاقة بين السياسة الضريبية والتوزان الاقتصادي.

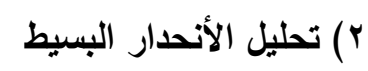

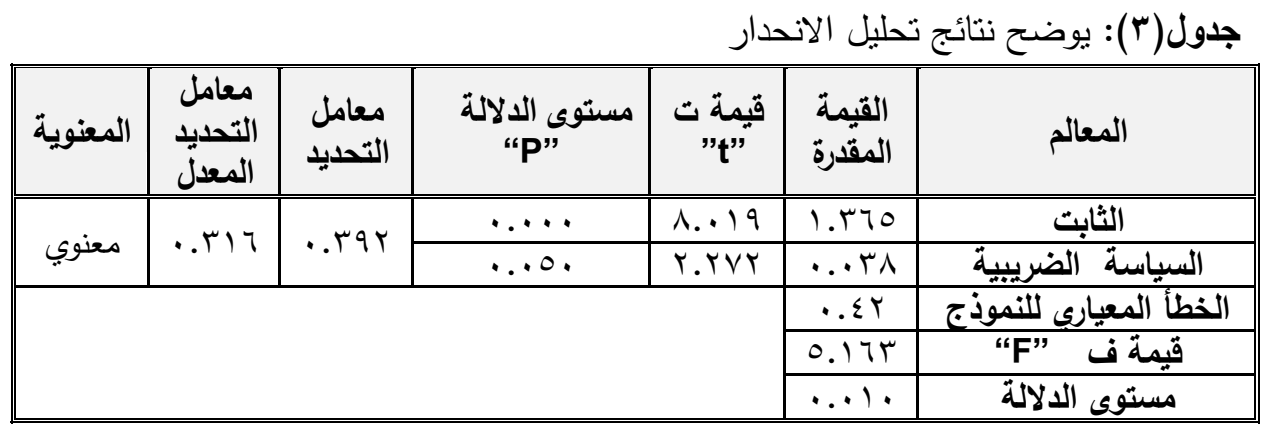

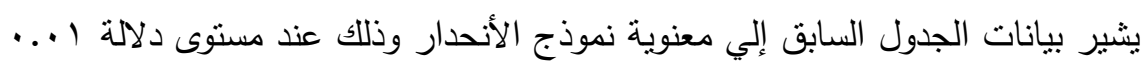

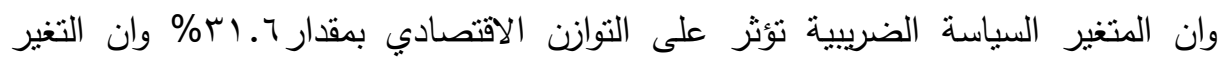
الحادث في التوزان الاقتصادي بمقدار الوحدة يسبيه التغير في السياسة الضريبية

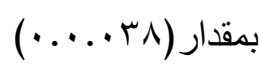


ثانيا: اختبار صحة فرض الدراسة الثاني توجد علاقة بين السياسة الضريبية وتحقيق التوزان البيئي: للتحقق من صحه الفرض قامت الباحثة بأستخدام معامل الارتباط البسيط

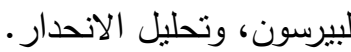
(1) معامل الارتباط البسيط لبيرسون جدول( ؛): يوضح العلاقة بين السياسة الضرييية والنوازن البيئي

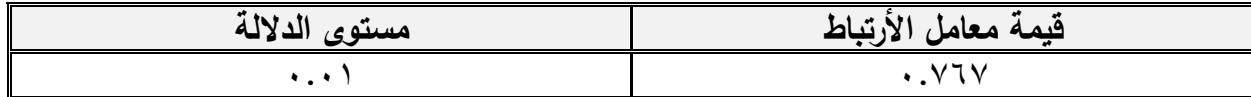

يوضح من بيانات الجدول السابق وجود علاقة ارتباط دالة أحصائياً بين السياسة الضريبية والتوازن البيئي حيث بلغت قيمة معامل الارتباط بينهما (VTV . . ) عند مستوى دلالة ا...، وهذا يدل على جوده علاقة بين السياسة الضريبية والتوزان البيئى، ولذلك هيتم عمل تحليل لمعامل الانحدار البسيط لمعرفة نوع العلاقة بين السياسة الضريبية والتوزان البيئى.

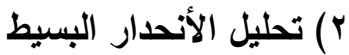
جدول رقم(•): يوضح نتائج تحليل الانحدار

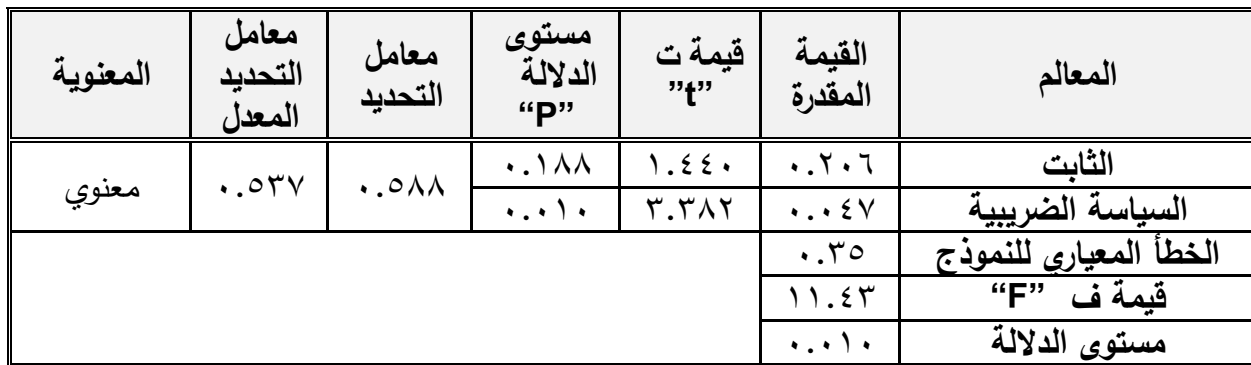

يوضح بيانات الجدول السابق إلي معنوية نموذج الانحدار وذلك عند مستوى دلالة ا... وإن المتغير السياسة الضريبية تؤثر على التوازن البيئي بمقدار V.ro\% وان التغير الحادث في التوزان البيئي بمقدار الوحدة يسببه التغير في السياسة الضريبية

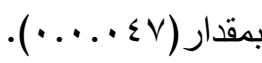




\section{النئائي التراسلة}

نتائج ذات علاقة بالاقتصاد:

( ) توجد علاقة أرتباط دالة أحصائياً بين السياسة الضريبية والتوازن الاقتصادي، وحيث بلغت قيمة

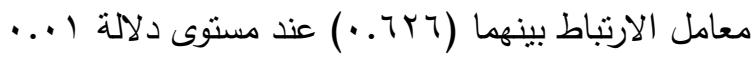

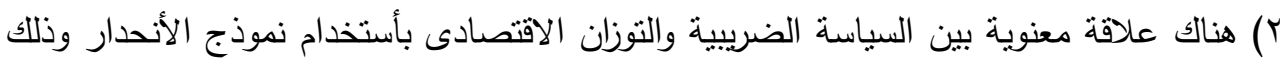

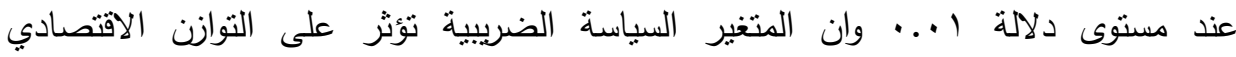

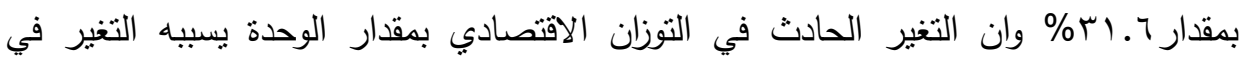

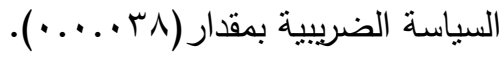

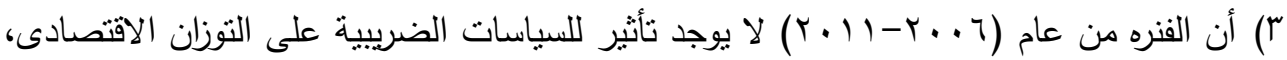

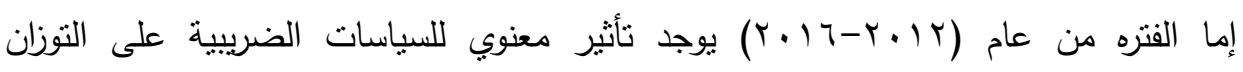
الاقتصادى

\section{نتائج ذات علاقة بالبيئة}

1) وجود علاقة أرتباط دالة أحصائياً بين السياسة الضريبية والنوازن البيئي حيث بلغت قيمة معامل

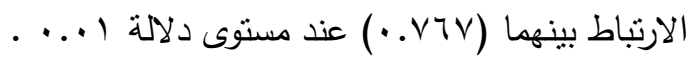

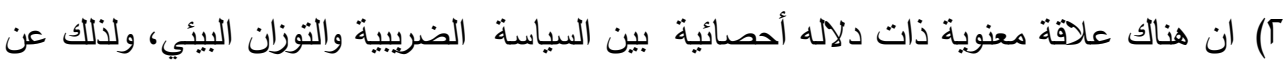
طريق أستخدام تحليل لمعامل الأنحدار وذلك عند مستوى دلالة الـ ل...، وإن المتغير السياسة الضريبية تؤثر على التوازن البيئي بمقدار V.ror\% وان التغير الحادث في التوزان البيئي بمقدار

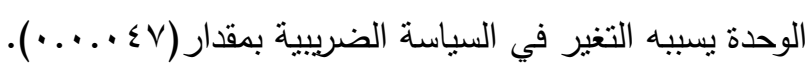

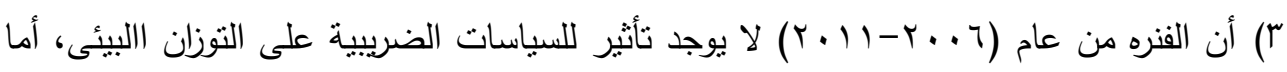

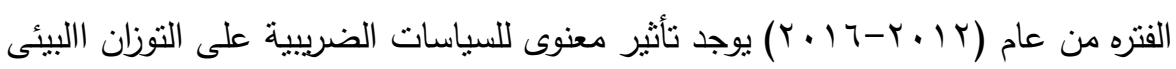
ع) الاعفاءات الضريبية دورا فعال فى الحفاظ على البيئة وذلك لتجنب التلوث قبل حدوثة واخذ بمبدا الوقاية خير من العلاج. 


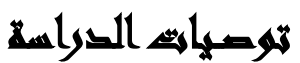

في ضوء النتائج السابقة ان نقترح عدداً من التوصبات التى قد تكون مفيدة فى مجال الدراسةقويمكن تقميها الى

\section{توصيات قصيرة الأجل}

() يجب العمل والتطوير المستمر لأجهزة القائمة بتنفيذ السياسة البيئية عن طريق تدريب العاملين بها وتوفير الاجهزة والمعدات للازمة للقيام بأعمالهم. r) توجية الإعفاءات الضربييةالموجوده بقانون الضرائب المصرية للأهتمام بالبعد البيئي لأنه لايقل أهمية عن الأبعاد الاقتصادية والاجتماعية التي تتمتع بكافه الإعفاءات الضريبية.

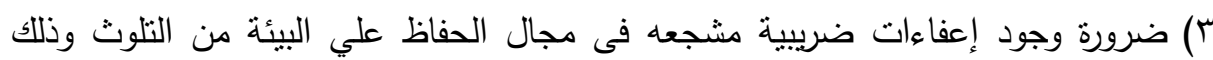
لتجنب أضرار أقتصادية وأجتماعية وصحية نتيجة التلوث من الافضل تجنب الفئه المشكلة قبل وقوعها أى تجنب النلوث قدر الأمكان وذللك لتشجيع للحفاظ علي البيئة. توصيات طويلة الأجل () ضرورة تغليظ العقوبات القانونية علي محدث التلوث واعتبار تلوث البيئة جناية وان تجمع العقوبات بين السجن والغرامة حتى تكون رادعة وتؤدى الى الأهداف المطلوبة .

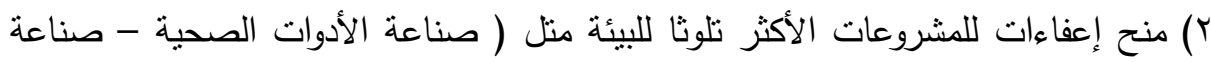

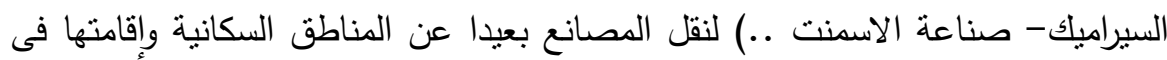
مناطق النائية أو الصحراء لتقليل التلوث النانج عنها.

\section{المراليج}

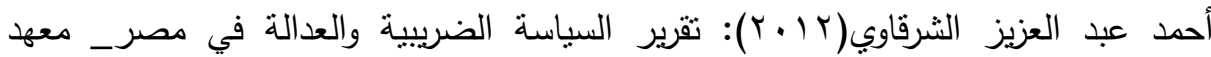
التخطيط القومي في مصر

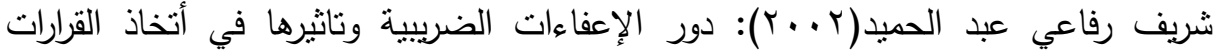

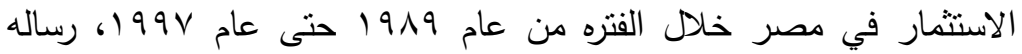

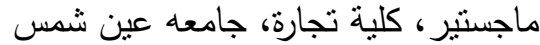
عبد العزيز سعيد( ... ب): النظم الضريبية مدخل تحليلي مقارن، الدار الجامعية، الاسكندرية 380

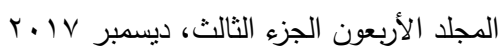




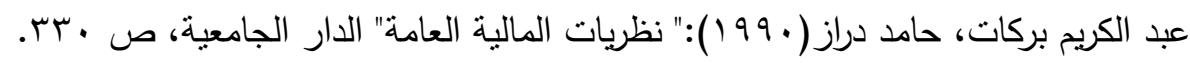

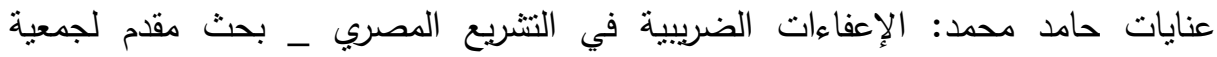

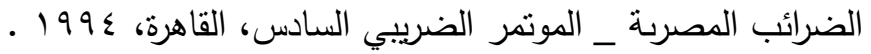

محمد رضا عدل(919 (1)): كتاب مبادى السياسة المالية والنقدية، مكتبة عين شمس، القاهرة، 1919

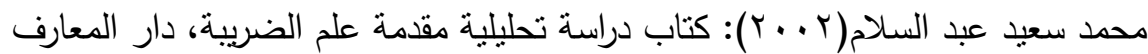

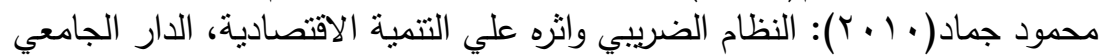

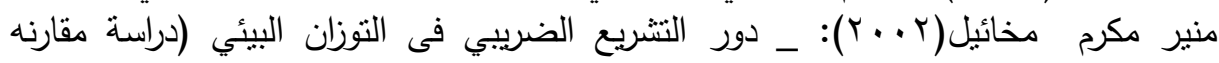

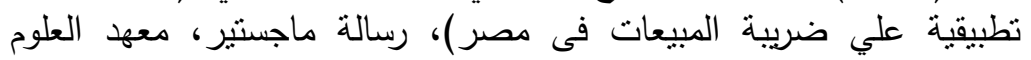

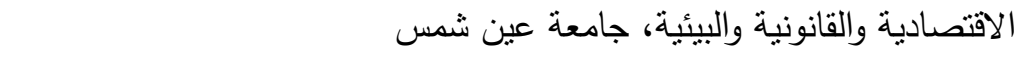

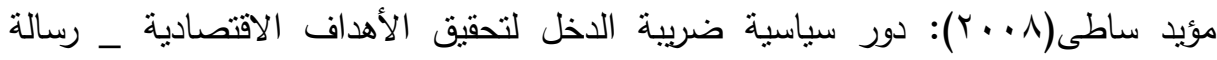
ماجستير _ _ جامعة فلسطين.

Bernard bobe_et piere llad fiscalité ET choix économmique inprimerie aubin_France _ 1978.

Gravelle Jane _ The Enron Debacle: Lessons For Tax Policy "Tax policy Center Discussion _ papers No.6_ February _ 2014

Monzer Kanf, Taxation Policy in an Islamic economy in fiscal policy and resource allocation ", Jeddan university, 1997, p147.

http://mawdoo3.com 
مجلة العلوم البيئية

معهذ الدراسات والبحوث البيئية - جامعة عين شمس البئ

\title{
IMPACT OF TAX POLICIES TO ACHIVEVE \\ ECONOMIC AND ENVIRONMENTAL BALANCE: \\ A COMPARATIVE STUDY
}

\author{
Abdelbar, A. H. ${ }^{(1)}$; Abdelbasset, W. F. ${ }^{(1)}$ \\ and Abd Elhafez, Marwa, A. \\ 1) Faculty of Commerce, Ain Shams University
}

\begin{abstract}
The problem of the study is that the tax policy that generates the return of the state from projects that pollute the environment is an important place among the other policy and it is one of the tools of fiscal policy that plays the greatest role in accomplishing the multiple objectives, thanks to its various tools which are one of the most important economic policy instruments in achieving economic development And the elimination of the problems that impede economic and environmental imbalance, and here explains the main problem of the study, which is the impact of the tax policy that is implemented on projects that pollute to achieve economic and environmental balance.

The main objective of the study is to study the impact of tax policy to achieve economic and environmental balance. This objective is achieved through a set of sub-objectives: knowledge of the definition of tax policy, knowledge of the impact of tax policy on economic and environmental balance and knowledge of tax exemptions and their importance and types.It is evident that from (2006 to 2007) to (20102011) there is no effect of tax policies on economic balance.

In order to achieve the objective of the study, the researcher adopted the scientific method in conducting a theoretical study and comparison by collecting actual data from the General Company for Ceramic and Chinese Tools (sanitary ware factory) in order to test the study hypotheses, analyze the data and interpret the results.
\end{abstract}


The study hypotheses included:

The first hypothesis: There is a significant relationship with statistical significance between tax policy and economic balance.

Second hypothesis: There is a significant correlation between tax policy and environmental balance.

The following results have been connected

- From 2006 to 2011 there is no effect of tax policies on economic balance. The period from 2012 to 2016 has a statistical effect on tax policy on economic balance.

- From 2006 to 2011 there is no impact of tax policies on urban balance, and the period from 2012 to 2016 has a statistical effect on tax policies on the environmental balance.

In the light of the previous findings, the study recommended a number of recommendations, the most important of which are:

- The components of tax policy should be designed in the light of the relations of coherence and interdependence between its parts and must balance justice, effectiveness and simplicity.

- Continuous work should be carried out on the continuous development of the institutions implementing environmental policy by training their personnel and providing equipment and equipment for the crisis to carry out their work.

- The existence of tax exemptions encouraging in the field of environmental protection from pollution in order to avoid economic, social and health damage caused by pollution. It is best to avoid the problem before it occurs, avoiding pollution as much as possible, in order to encourage the preservation of the environment. 